\title{
A CHARACTERIZATION OF THE UNIQUELY ERGODIC ENDOMORPHISMS OF THE CIRCLE
}

\author{
LLUÍS ALSEDÀ, FRANCESC MAÑOSAS, AND WIESLAW SZLENK
}

(Communicated by Charles Pugh)

\begin{abstract}
We characterize the uniquely ergodic endomorphisms of the circle in terms of their periodic orbits.
\end{abstract}

Let $f: \mathbf{S}^{1} \rightarrow \mathbf{S}^{1}$ be a continuous endomorphism of the circle $\mathbf{S}^{1}$. Denote by $F: \mathbf{R} \rightarrow \mathbf{R}$ its lifting to the universal covering space. Then, for all $x \in \mathbf{R}$, $F$ satisfies $F(x+1)=F(x)+k$ for some $k \in \mathbf{Z}$. The number $k$ is called the degree of $f$ (when necessary this number will also be called the degree of $F$ ). Denote by $\mathscr{L}$ the class of all liftings of continuous endomorphisms of the circle and by $\mathscr{L}_{1}$ the class of maps from $\mathscr{L}$ having degree 1 .

Let $F \in \mathscr{L}_{1}$. For each $x \in \mathbf{R}$ we define the rotation number of $x$, denoted by $\rho_{F}(x)$, as (see [NPT]) $\lim \sup _{n \rightarrow \infty} F^{n}(x) / n$.

From [I] it follows that the set $L_{F}=\left\{\rho_{F}(x): x \in \mathbf{R}\right\}$ is a closed interval (or perhaps a point). This interval is called the rotation interval of $F$. In what follows, whenever $L_{F}$ degenerates to a point it will be denoted by $\rho(F)$. In such a case we shall talk about the rotation number of $F$. It is well known that when $F$ is nondecreasing, it has degenerate rotation interval.

A continuous endomorphism of the circle $f$ is said to be uniquely ergodic if there exists a unique $f$-invariant probability measure on $\mathbf{S}^{1}$. From $[\mathrm{H}]$ it follows that every homeomorphism of the circle of degree one with irrational rotation number is uniquely ergodic. The aim of this paper is to extend this result to the endomorphisms of the circle. In fact, we shall give necessary and sufficient conditions for an endomorphism of the circle to be uniquely ergodic. Our main result is the following

Theorem. A circle endomorphism $f$ is uniquely ergodic if and only if it has at most one periodic orbit.

To prove the above theorem we shall use the following results. For them we have to introduce some notation.

Received by the editors January 16, 1991 and, in revised form, June 24, 1991.

1991 Mathematics Subject Classification. Primary 34C35, 54H20.

Key words and phrases. Circle maps, periodic orbits, uniquely ergodicity.

Supported by DGCICYT grant number PB86-0351.

This paper was written while W. Szlenk was a visiting Professor at the Universitat Autonoma de Barcelona. 
We shall say that a point $x \in \mathbf{R}$ is periodic $(\bmod 1)$ of period $q$ for a map $F \in \mathscr{L}$ if $F^{q}(x)-x=p$ for some $p \in \mathbf{Z}$ and $F^{i}(x)-x \notin \mathbf{Z}$ for $i=1, \ldots, q-1$. Clearly, if $F$ is a lifting of $f$ then $x$ is periodic $(\bmod 1)$ for $F$ if and only if $e^{2 \pi i x}$ is periodic for $f$ and their periods are equal. For $x \in \mathbf{R}$ the set $\left\{F^{n}(x)+m: n \geq 0\right.$ and $\left.m \in \mathbf{Z}\right\}$ will be called the $(\bmod 1)$ orbit of $x$. The $(\bmod 1)$ orbit of periodic $(\bmod 1)$ point will be called a periodic $(\bmod 1)$ orbit. We note that if $x$ is a periodic $(\bmod 1)$ point of a map $F \in \mathscr{L}_{1}$ and $F^{q}(x)=x+p$, then $\rho_{F}(x)=p / q$.

Proposition 1. The following statements hold:

(1) For each $a \in \mathbf{R} \backslash \mathbf{Q}$ there exists a noninvertible map $F \in \mathscr{L}_{1}$ such that $\rho(F)=a$.

(2) For each noninvertible map $F \in \mathscr{L}_{1}$ with $\rho(F) \notin \mathbf{Q}$ there exists an $F$ invariant Cantor set $C \subset \mathbf{R}$ such that $\left.F\right|_{C}$ is semiconjugate to the rotation by angle $\rho(F)$. Moreover, for each $x \in C$, the $(\bmod 1)$ orbit of $x$ is dense in $C$.

Proof. (1) Given $a \in \mathbf{R} \backslash \mathbf{Q}$, by the Denjoy construction (see [D, N]) there exists a diffeomorphism $G \in \mathscr{L}_{1}$ with $\rho(G)=a$. Moreover, the set $\mathbf{R}$ can be decomposed into a disjoint union of a $G$ invariant open set $I$ and a $G$ invariant cantor set $C$. Let $K$ be a connected component of $I$ and assume that $K \subset[0,1]$. Set $L=G(K) \subset I$. Clearly there exists a map $F \in \mathscr{L}_{1}$ such that

(i) $\left.F\right|_{[0,1] \backslash K}=\left.G\right|_{[0,1] \backslash K}$,

(ii) $F(K)=L$,

(iii) $\left.F\right|_{K}$ is noninvertible.

To end the proof of (1) it only remains to show that $\rho(F)=\rho(G)=a$.

Since $\rho(G) \notin \mathbf{Q}, G$ has no periodic points. Thus $\left.F\right|_{C}$ has no periodic orbits. Since $G^{n}(K) \cap(K+m)=\varnothing$ for all $n>0$ and $m \in \mathbf{Z}$, by construction the same holds for $F$. Therefore, $F$ has no periodic points. By Misiurewicz's Theorem (see [M1]) we have the $L_{F}$ degenerates to an irrational. On the other hand, since $\left.F\right|_{C}=\left.G\right|_{C}$, we get that $a \in L_{F}$. So $\rho(F)=a$.

(2) Let $F \in \mathscr{L}_{1}$ be a noninvertible map with $\rho(F)=a \notin \mathbf{Q}$. From Theorem A of [M2] it follows that there exists a closed, nonempty set $C$ that is invariant, minimal (i.e., for each $x \in C$ the $(\bmod 1)$ orbit of $x$ is dense in $C$ ) and such that $\left.F\right|_{C}$ is semiconjugate to a rotation (of course, by angle $\rho(F)$ ). Moreover, $C$ is either $\mathbf{R}$ or a Cantor set. Therefore, $\left.F\right|_{C}$ is nondecreasing. Since $F$ is noninvertible, we get that $C \neq \mathbf{R}$. This ends the proof of the proposition.

Remark 2. From Proposition 1(1) it follows that there exist noninvertible endomorphisms of the circle with irrational rotation number. Therefore, the theorem really extends Herman's result to noninvertible endomorphisms of the circle.

Proposition 3. If a continuous endomorphism of the circle $f$ has only one periodic orbit $P$ then the $\Omega$-limit set of $f$ is $P$.

Proof. Note that if a continuous endomorphism of the circle $f$ has degree different from $-1,0$, and 1 then it has infinitely many periodic orbits. Also, if $f$ has degree -1 it has at least two fixed points. Hence, if $f$ has only one periodic orbit then the degree of $f$ must be 1 or 0 . Let $F$ be a lifting of $f$. 
Assume that the degree of $f$ is 1 . In view of [M1], for each rational number $p / q \in L_{F}$ with $p$ and $q$ relatively prime, there exists a periodic $(\bmod 1)$ orbit of $F$ with period $q$ and rotation number $p / q$. Therefore, since $f$ has only one periodic orbit, $L_{F}$ has to be degenerate. On the other hand, since $f$ has a periodic orbit, we have $L_{F} \cap \mathbf{Q} \neq \varnothing$. Then $L_{F}=\{p / q\}$ for some $p \in \mathbf{Z}$ and $q \in \mathbf{N}$, with $p$ and $q$ relatively prime, and the period of $P$ is $q$. Set $\mathscr{P}=\left\{x \in \mathbf{R}: e^{2 \pi i x} \in P\right\}$. Clearly $\mathscr{P}$ is the only periodic (mod 1 ) orbit of $F$. Denote the map $F^{q}-p$ by $G$. Clearly $G(x)=x$ for each $x \in \mathscr{P}$, and these are the only periodic $(\bmod 1)$ points of $G$. Without loss of generality we may assume that $0 \in P$. Then, since $G \in \mathscr{L}_{1}$, we get that $G(1)=1$, and hence $G([0,1]) \supset[0,1]$. Thus $G^{n}([0,1]) \subset G^{n+1}([0,1])$ for all $n \in \mathbf{N}$. Let $K$ be the closure of $\bigcup_{n=1}^{\infty} G^{n}([0,1])$. Clearly, $K$ is a $G$-forward invariant closed interval containing $[0,1]$. If $K$ is not bounded, then there exist $z \in[0,1]$ and $m \in \mathbf{N}$ such that $G^{m}(z)>z+1$. Therefore, $G$ has a periodic $(\bmod 1)$ point of period $m$ and rotation number $1 / m \neq 0$, which is a contradiction. Thus $K$ is bounded (in fact, $K \subset[-1,2]$ ). Since the interval map $\left.G\right|_{K}$ has a finite number of periodic points (namely, $\mathscr{P} \cap K$ ), it follows from [S1] (see also [S2]) that the $\Omega$-limit set of $\left.G\right|_{K}$ is $\mathscr{P} \cap K$. For each $x \in \mathbf{R}$ there exists $r \in \mathbf{Z}$ such that $x-r \in K$. Since $G \in \mathscr{L}_{1}$, we get that $G^{n}(x-r)=G^{n}(x)-r$ for all $n \in \mathbf{N}$. Therefore, the $\Omega$-limit set of $G$ is $\mathscr{P}$.

Assume now that $f$ has degree 0 . Since $f$ has at least a fixed point, the only periodic point of $f$ is a fixed point. Therefore, $F$ has a unique periodic $(\bmod 1)$ orbit $\mathscr{P}$ that has period 1 . Since $f$ has degree 0 , we have that $F(x+m)=F(x)$ for all $x \in[0,1]$ and $m \in \mathbf{N}$. Hence, there exist $z \in \mathscr{P}$ such that $F(\mathscr{P})=z$. Therefore, $z$ is the unique fixed point of $F$. Set $K=F([0,1])$. Clearly $F(\mathbf{R})=K$ and $z \in K$. Since the $\Omega$-limit set of $F$ coincides with the $\Omega$-limit set of the interval map $\left.F\right|_{K}$, we only have to show that $z$ is the $\Omega$-limit set of $\left.F\right|_{K}$. This can be done by using the same result as in the case of degree 1 .

Proof of the theorem. Note that if a continuous endomorphism of the circle $f$ has two periodic orbits then it is not uniquely ergodic since invariant measures exist concentrated on each of the two orbits. This proves the "only if" part.

Suppose that $f$ has no periodic orbits, and let $F$ be a lifting of $f$. Then, in view of Lemma 4 and Theorem 2 of [AK], for every $x \in \mathbf{R}$, the $\Omega$-limit set of $x$ is equal to $C$, where $C$ is as in Proposition 1 . Thus every $F$-invariant probabilistic measure is concentrated on $C$. In view of Proposition $1,\left.F\right|_{C}$ is semiconjugate to a rotation of the circle by an irrational angle. So $F$ is uniquely ergodic. In the remaining cases, from Proposition 3 , we have that the nonwandering set of $f$ is the periodic orbit. Therefore, again there is only one probabilistic $F$-invariant measure.

Remark 4. From the proof of the theorem it follows that for an endomorphism $f$ to be uniquely ergodic there are only three possibilities:

(1) $f$ has degree one and irrational rotation number;

(2) $f$ has degree one, rational rotation number $p / q$ with $p$ and $q$ relatively prime and a unique periodic orbit with period $q$;

(3) $f$ has degree zero and a unique periodic orbit that is a fixed point. 


\section{ACKNOWLEDGMENTS}

The authors feel indebted to the editor and the referee of this paper for valuable remarks.

\section{REFERENCES}

[AK] J. Auslander and Y. Katznelson, Continuous maps of the circle without periodic points, Israel J. Math. 32 (1979), 375-381.

[D] A. Denjoy, Sur les courbes definies pour les equations differentielles a la surface du tore, J. Math. Pures et Appl. 7 (1932), 333-375.

[H] M. Herman, Sur la conjugaison differentiable des difféomorphismes du cercle a des rotations, Inst. Hautes Études Sci. Publ. Math. 49 (1979), 5-234.

[I] R. Ito, Rotation sets are closed, Math. Proc. Cambridge Philos. Soc. 89 (1981), 107-111.

[M1] M. Misiurewicz, Periodic points of maps of degree one of a circle, Ergodic Theory Dynamical Systems 2 (1981), 221-227.

[M2] _ , Twist sets for maps of the circle, Ergodic Theory Dynamical Systems 4 (1984), 391404.

[N] Z. Nitecki, Differentiable dynamics, M.I.T. Press, Cambridge, MA, 1971.

[NPT] S. Newhouse, J. Palis, and F. Takens, Stable families of dynamical systems I, Publ. Math. 57 (1983), 5-72.

[S1] A. N. Sharkovskiî, On some properties of discrete dynamical systems, Proc. of Sur la Théorie de l'Iteration et ses Applications, vol. 332, C.N.R.S., Paris, 1982, pp. 153-158.

[S2] A. N. Sharkovskiǐ, et al., Dinamica odnomiernyh otobraženi, Naukova Dumka, Kiev, 1989.

Department de Mathemàtiques, Universitat Autònoma de Barcelona, 08193 BellaTERra, Barcelona, SPAin

E-mail address: IMAT0@CC.UAB.ES

Departament de Matemàtica Aplicada II, E.T.S. d'Enginyers Industrials de Terrassa, Universitat Politècnica de Catalunya, Colom 11, 08222 Terrasa, Barcelona, Spain

Institute of Applied Mathematics and Mechanics, University of Warsaw, KrakowsKie Przedmiescie 26-28, Warsaw, Poland

E-mail address: SZLENK@PLEARN.BITNET 